\title{
Editorial
}

\section{Pothunter or Professional?}

\author{
DAVID A. ARMOUR, Editor
}

"Pothunter." The very word is anathema to all professional archaeologists. We apply the designation "pothunter" to anyone who digs a site simply for the thrill of the dig and the relics which he collects. The excavator probably learns something from the experience, and he does collect and preserve the artifacts which interest him. However, the "pots" repose in his basement, his memory fades with regard to the context from which they were ripped, and the site has been destroyed beyond repair. If his work has been thorough, no one in the future can ever excavate the site again.

We, as professionals, are all aware of the destructive nature of archaeology, and yet we are often no better than the pothunter. True, when we excavate a site, we apply all the professional procedures. We lay out a grid and excavate in a systematic fashion. We record our data on square sheets, field notes, and film, and we meticulously place each fragment of an artifact in precisely recorded sacks. Our professionalism dictates that we carefully excavate according to the standards of our training. Usually we also enjoy the work and are thrilled by the discovery of an exciting artifact or structure despite the drudgery of the meticulous record keeping.

At the end of the dig, we leave confident that we have salvaged every last scrap of evidence. We store our artifacts and file our notes. When we get time, we intend to start our analysis and write the final report.

Then we run out of steam. Sometimes we work on for some time due to a contract's pressure to produce a report, or we are driven by the intrinsic interest of the site. However, all too often we get caught up in other activities. While the excavation nags at our conscience, the artifacts get misplaced, mixed up, or lost, our notes get mislaid, and the synthesizing data we carry in our heads gradually fades. New, exciting sites cry to be dug, and the job we never completed is relegated to obscurity.

As a result, the site is destroyed, the artifacts are scattered, and no one is the wiser and they never can be.

Perhaps I have painted an exceptionally gloomy picture, but I am afraid I have not. Each of us, I am certain, can think of many similar examples. My basic plea is this: If a site is excavated, it must have a report written about it. Because archaeological excavation, whether by pothunter, amateur, or professional, is totally destructive, the responsibilities of the researcher are much greater than almost any other discipline. All that remain after a dig are the destroyed site, a collection of artifacts, the exca- 
vator's memory, any pictures or notes he took, and a report if one was written. No one can redig the site to redo the experiment. An archaeologist is like an historian who, as he studies the manuscript archives, tears up each page as he reads it. This, of course, does not happen, and we would be horrified if it did. When a sociologist, historian, or anthropologist receives a grant to conduct research and after months or years fails to produce a report, the money has only been wasted. Usually the problem has not been destroyed. If funding can be obtained, it is feasible for someone else to redo the research and solve the problem.

How does it happen that professional archaeologists who realize the imperative to write final reports often get sidetracked? One cause is faulty planning. No project, unless it is a strictly salvage operation, should be begun unless it has been properly conceived, from the beginning of excavation to the publication of the final report. Often in planning an archaeological project one thinks only of the time and expense of the dig itself. Yet we all know, as J. C. Harrington pointed out in Archaeology and the Historical Society (1965:42), that one should spend from two to six months analyzing and writing for every month spent in the field. Furthermore, if historical research is also required, that time must be added. How many of us really budget that much time and money for the project once digging stops? Yet if we are not to be simply pothunters, we must analyze the data and write the final report.

For example, if we receive $\$ 5,000$ for a project, we should probably allocate only $\$ 1,000$ for excavation and $\$ 4,000$ for analysis and publication. In writing grant proposals, however, we normally shy away from including such large sums for analysis and weight the funds toward excavation. We do this because of the popular identification of archaeology only with excavation and not with the laborious and timeconsuming processing and analyzing of the data and the writing and publication of the final report.

Let us repeatedly ask ourselves what is really the goal of excavation. Is it simply the excitement of the dig? Is it the collection of artifacts? Or is it the solving of an important archaeological problem? If it is the latter, it can only be adequately achieved by a written or published report. With the report as our main goal, we should carefully structure our entire research effort toward achieving that end. Without the report, the whole project is a waste of time, money and irreplaceable cultural resources.

A second reason for our lack of published output stems from the fact that most professionals are also teachers. Consequently we have our summers free to excavate, but the nine months of the academic year do not allow sufficient time to analyze the data from the summer's dig. We never quite catch up because we hate to pass up a summer digging season. We professionals should spend less time digging and more time analyzing and writing.

A third cause for failure to write a report or to publish is fear. We realize the complexity of the material culture which we have excavated, so we decide to forego a final report until we have found all the answers. The result is we never write the report. Fear of criticism by supposedly better informed colleagues stifles our efforts and blocks our presentation of data.

At this point in time historical archaeology desperately needs the publication of records of recently conducted excavations. These reports will not be definitive, as if any report ever is. Yet they will make possible a body of information which others can use. Practitioners of historical archaeology desperately want to know what others 
are finding in order to better assess the artifacts they have found. Only when you sit down to write a report do you realize how little comparative data there is in print.

During the past two years the Society for Historical Archaeology has taken steps to meet the need. By means of the Newsletter we have attempted to provide information about who was conducting excavations and what type of sites were being investigated. The annual publication, Historical Archaeology, is a vehicle where the results of historical archaeology research may be published. Furthermore, in Historical Archaeology John Cotter has brought together a list of recent publications in the field so that we can know what others are doing. Two things have struck me as editor. One is how few manuscripts are sent to me for possible publication, and the other is the shortness of Cotter's bibliography. In contrast, the Newsletter lists numerous excavations in progress throughout the country. I can only conclude that our profession is digging widely but is not taking the time to analyze and publish.

Some of us are frequent conference attenders, and we hear numerous exciting presentations. Yet few of them ever appear in print, and soon we forget what we heard. Papers read at a conference are no substitute for publication since few people hear them and, too often, little is remembered. Only publication forms a useable permanent record.

In this vein let me emphasize the importance of a published photographic record of the structures and artifacts. Words are often so inadequate, particularly when we are not quite certain what we are describing. Yet good clear photographs or drawings presented with a scale will go a long way in helping another researcher. The artifacts will be particularly valuable if they came from a closely dated site. The profession is still groping for good chronological indicators, and, only when sufficient data has been accumulated from large numbers of sites, will we be able to tell when certain objects were in popular use and not simply when they were manufactured.

Besides the need for the publication of relatively raw archaeological data, we also need people who are experts on some particular artifact or culture to analyze and synthesize the data unearthed by field archaeologists. In many cases these experts may never or seldom excavate, but they can provide the profession with studies which will be of eminent value for the digging archaeologists. Yet here again it is almost impossible for someone to bring together a great synthesis unless we provide him with the published data he needs.

If you do complete your final report, two courses of action are possible. One is to go no further, simply file the report and forget it. If you take this approach, you do fulfill the major part of your professional obligation. However, I urge you to send a copy of the report to John Cotter, National Park Service, Northeast Region, 143 South Third Street, Philadelphia, Pennsylvania 19106, so that he can list it in his bibliography. Then anyone interested in your site can correspond directly with you and perhaps secure a copy of your report.

Should you decide to publish, there are several avenues open. If the site is not too extensive, you may be able to publish your report in a state or local archaeological publication or it may find a place in Historical Archaeology. Should the report be book length, problems are more acute, due to the greater cost involved. Here a careful program budget is invaluable, for funds would have been allocated from the beginning to publish the report. Otherwise, it is most difficult to obtain publication funds for a major report. However, it is often possible and extremely valuable to pub- 
lish portions of the research separately, for instance, studies of certain artifact types or individual structures within a complex site.

In conclusion it should become an axiom in historical archaeology that, if one digs, he must write, and, if one writes, he should publish. That is what ultimately separates the professional from the "pothunter." 